\title{
(2) \\ Osteometría de Vicugna vicugna Molina, 1782 en el Pleistoceno final de Patagonia meridional chilena: Implicancias paleoecológicas y biogeográficas
}

RMA

Dossier - Arqueología

\author{
1Programa de Doctorado, Facultad de Ciencias Sociales, UNCPBA, Argentina. \\ E-mail: r.labarca.e@gmail.com \\ ${ }^{2}$ Centro de Estudios del Hombre Austral, Universidad de Magallanes, Chile. \\ E-mail: alfredo.prieto@umag.cl
}

\begin{abstract}
Resumen
Se presenta un análisis osteométrico uni y multivariado con los materiales fósiles -principalmente huesos cortos y falanges- de Vicugna sp. del yacimiento Cueva Lago Sofía 4 (13500 - 11000 AP), ubicado en la provincia de Última Esperanza (Región de Magallanes, Chile). Los resultados indican que los elementos analizados poseen un tamaño compatible con los registrados para Vicugna vicugna, no superando en ningún caso a los especímenes conocidos para Vicugna gracilis del Pleistoceno de la región Pampeana. Esto confirma la información morfológica y de ADN antiguo que indicaba la presencia de Vicugna vicugna en la Patagonia meridional chilena. Se discuten los resultados en términos taxonómicos, biogeográficos y paleoecológicos sugiriendo que la presencia de este taxón fuera de su rango actual de distribución se debió a las condiciones ambientales compartidas entre la Puna y Patagonia austral durante la transición Pleistoceno-Holoceno. La extinción de V. vicugna se habría debido al impacto de los cambios climáticos ocurridos al inicio del Holoceno producto de su dieta pastadora y etología no migratoria y territorial, a lo que se habría sumado una caza por parte de depredadores humanos y no humanos.
\end{abstract}

Palabras clave: género Vicugna, osteometría, Pleistoceno, Patagonia

Osteometry of Vicugna vicugna Molina, 1782 in the late Pleistocene of southern chilean Patagonia: palaeoecologic and biogeographic implications.

\begin{abstract}
This contribution presents the results of single and multi-variable osteometric analyses performed on a sample of short bones and phalanxes assigned to Vicugna sp. from Lago Sofía Cave 4 site (13500 - 11000 BP), Región de Magallanes, Chile. The elements analyzed show a similar size to the reference collections for Vicugna vicugna, and do not exceed the metrics for the pampean pleistocenic specimens of Vicugna gracilis. This confirms the morphological and ancient DNA data that indicated the presence of vicuña in southern Chilean Patagonia. The taxonomic, biogeographic and palaeoecologic discussion of these results, suggests that the presence of this species outside its current distribution range was due to shared environmental conditions between the Puna and Patagonia during the Pleistocene-Holocene transition. The extinction of this form is, most likely, due to the impact of climatic change during the begining of the Holocene over its pasturing diet and its territorial and non migratory habits. It is added to this, a hunting pressure by human and non-human predators.
\end{abstract}

Keywords: Vicugna genus, osteometry, Pleistocene, Patagonia

La familia Camelidae presenta en América y en la actualidad cuatro formas vivientes, dos silvestres (Lama guanicoe Müller, 1776 y Vicugna vicugna Molina, 1782) y dos domésticas (Lama glama Linnaeus, 1758 y Vicugna pacos Linnaeus, 1758) (Franklin 1982; González et al. 2000; Marín et al. 2007; Wheeler 1995). En el Plio-Pleistoceno sudamericano, no obstante, habría existido una mayor diversidad de taxones (por lo menos cuatro géneros y cerca de doce especies, Guerin y Faure 1999; Hoffstteter 1952; Menegaz 2000; Menegaz y Ortíz-Jaureguízar 1995), aún cuando el número exacto de formas extintas no está del todo definido. Gran parte de esta incertidumbre taxonómica se debe a la similitud osteomorfológica observada al interior de la familia. En efecto, los elementos post craneales poseen un escaso valor diagnóstico en términos morfológicos, concentrándose casi la totalidad de los caracteres en el cráneo y particularmente en ciertas piezas dentales (p.e mayor o menor presencia de esmalte en los incisivos, raíces abiertas o cerradas de éstos, ausencia o presencia de endostilo en M2 y M3). La homogeneidad es tal, que algunos rasgos taxonómicos aún se encuentran en discusión (Cabrera 1932; Menegaz 2000; 
Tauber 1999). Una vía para solucionar esta dificultad es la aplicación de técnicas osteométricas, partiendo del supuesto de que existen diferencias significativas en los tamaños de las distintas formas de la familia (Cartajena 2002; Guerin y Faure 1999; Izeta 2004; Menegaz 2000; Menegaz et al. 1989). Con este criterio, es posible distinguir tres grandes grupos: camélidos pequeños (afines al género Vicugna), camélidos medianos (afines a Lama glama y Lama guanicoe) y camélidos grandes (con formas extintas como Hemiauchenia y Palaeolama).

Particularmente dentro del grupo de menor talla, se reconocerían por lo menos tres formas, la vicuña (Vicugna vicugna), restringida en la actualidad a sectores andinos por sobre los 3000 msnm, Vicugna provicugna Boule y Thévenin, 1920 del Pleistoceno de Tarija y Lama (Vicugna) gracilis Gervais y Ameghino, 1880 con registros en el área pampeana, patagónica argentina y posiblemente norchilena (García et al. 2008; Guerin y Faure 1999; López y Labarca 2005; Menegaz 2000; Menegaz et al. 1989; Miotti y Salemne 1999; Paunero et al. 2004; Tauber 1999). V. provicugna aparentemente correspondería a la forma de mayor tamaño del género (Guerin y Faure 1999), aunque no existen criterios diagnósticos claros. Lama (V.) gracilis fue originalmente descripta por Gervais y Ameghino (1880) como Auchenia gracilis, siendo posteriormente denominada Lama gracilis por López-Aranguren (1930). Su validez específica fue discutida por Cabrera (1932), quien la incluyó dentro de $V$. vicugna. Años más tarde Menegaz et al. (1989) y Menegaz (2000) revalidan esta forma, nombrándola Lama (Vicugna) gracilis, ya que estos autores incluían todas las formas vivientes de la familia Camelidae dentro de un solo género (Lama) con dos subgéneros, Lama y Vicugna. Recientes estudios genéticos (Kadwell et al. 2001; Marín et al. 2007), han confirmado la separación de los representantes actuales de la familia en dos géneros, por lo que Lama $(V$.) gracilis, debería denominarse Vicugna gracilis de acuerdo a las características afines con el genero Vicugna (Labarca en preparación). Esta propuesta taxonómica será utilizada a lo largo del presente trabajo.

En Patagonia chilena, Prieto y Canto (1997) describen por primera vez restos dentales de morfología compatible con el género Vicugna en los yacimientos finipleistocénicos de Tres Arroyos 1 (Tierra del Fuego) y Cueva Lago Sofía 4 (Última Esperanza). Las piezas fueron datadas por AMS en $10630 \pm 70$ AP y $13100 \pm 70$ AP, respectivamente (Weinstock et al. 2009). Las características mencionadas por éstos autores apuntaban hacia una relación más cercana con la actual vicuña que con su pariente extinto, V. gracilis (Prieto y Canto 1997). Recientemente, Weinstock et al. (2009), realizaron análisis de ADN antiguo de ambas piezas, concluyendo que se trata de Vicugna vicugna. Estos autores sugieren además, que los elementos asignados a $V$. gracilis en actual territorio argentino probablemente correspondan a $V$. vicugna.
La revisión de los materiales óseos de las antiguas excavaciones de Cueva Lago Sofía 4 (CLS-4), así como nuevas intervenciones estratigráficas realizadas por nosotros han arrojado una importante cantidad de restos post craneales que cualitativamente pudieron ser adscriptos a Vicugna sp. (Labarca 2008). Este trabajo presenta un análisis osteométrico exploratorio con los restos de CLS-4 (principalmente huesos cortos y falanges) con el objeto de discutir de manera cuantitativa esta primera aproximación taxonómica y examinar la posibilidad de asignar específicamente los materiales. Se aplicaron herramientas uni y multivariadas utilizadas previamente en trabajos arqueológicos y paleontológicos con restos de camélidos (p.e. Izeta 2004; Menegaz et al. 1989), utilizando medidas de referencia de Vicugna vicugna, Vicugna gracilis del

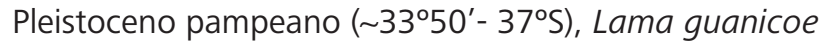
patagónicos y un ejemplar recuperado en las excavaciones del sitio arqueológico de Agua de la Cueva ( 32 $\left.50^{\prime} S\right)$, en donde se ha identificado una forma de menor tamaño que el guanaco actual (García et al. 2008).

\section{La Cueva Lago Sofía 4}

La Cueva Lago Sofía 4 se ubica en la Provincia de Última Esperanza (Región de Magallanes, Chile) a los pies del Cerro Mocho, al noreste del Lago Sofía, en una antigua terraza generada por el lago periglaciar Última Esperanza, a unos 120 msnm. Presenta una morfología subtriangular, con un largo máximo de unos 16 metros. En la línea de goteo exhibe unos tres metros de alto por seis de ancho. En su interior, la cueva pierde rápidamente sus dimensiones iniciales, ya que un talud rocoso de fuerte pendiente W-E genera un estrecho pasillo de acceso de no más de un metro de ancho ubicado en el sector Este de la cueva. Tras este pasillo, se ubica una antecámara de piso rocoso, la que desemboca en una cámara completamente oscura de unos seis metros de largo, por unos 2,5 de ancho y 1,5 de alto (Figura 1). La cueva se habría formado por la disolución de las capas de lutita intercaladas en la Formación Cerro Toro (Wellman 1972) y por desprendimiento de bloques del techo causado por el agua de escurrentía (Borrero et al. 1997).

Durante los años 1989, 1990 y 1993, entre otras actividades sistemáicas se excavaron un total de cuatro cuadrículas de $1 \mathrm{~m}^{2}$ (1C, 2C, 3C y 4C) en la cámara interior hasta una profundidad aproximada de $30 \mathrm{~cm}$ (Borrero et al. 1997). Se recuperaron un total de 2.829 restos óseos, a partir de los cuales se puedo identificar Lama guanicoe, Lama sp., Vicugna sp. Hippidion saldiasi Roth, 1899, Mylodon darwinii Owen, 1839, Hippocamelus bisulcus Molina, 1782, Conepatus humboldtii Gray, 1837, Myocastor coipus Molina, 1782, Lagidium viscacia Molina ,1782, Lycalopex culpaeus Molina, 1782 y Smilodon populator Lund, 1842 (Borrero et al. 1997, Labarca et al. 2008, Prieto y Canto 1997). Las modificaciones observables en la superficie de los restos óseos se relacionaron fundamentalmente con mordeduras generadas por el 


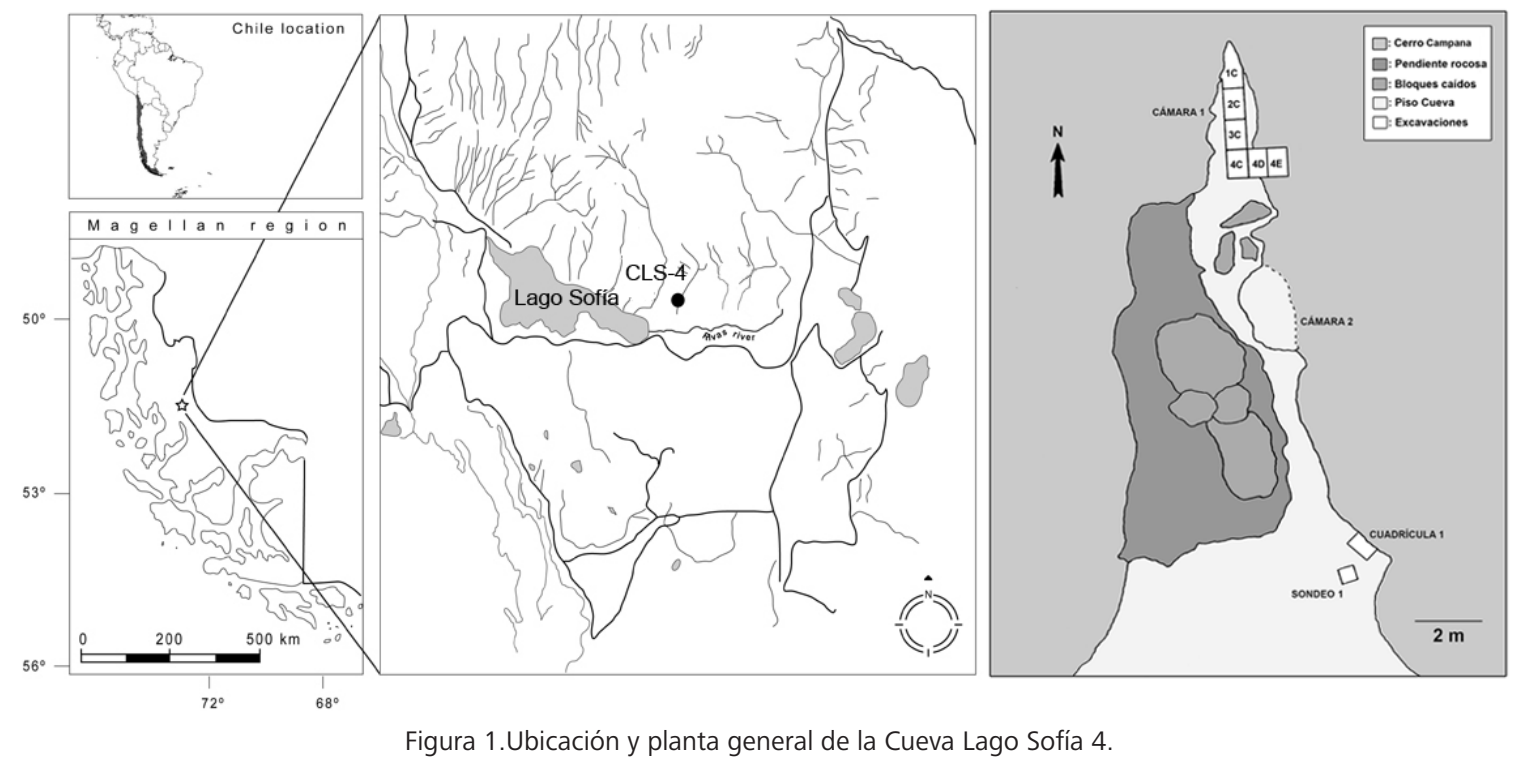

consumo de carnívoros (Borrero et al. 1997). De acuerdo a Borrero et al. (1997), la intensidad, frecuencia y morfología de los daños serían consecuentes con la acción de un gran felino, posiblemente Panthera onca mesembrina Cabrera 1934, sin descarte el aporte marginal de otros carnívoros menores (e.g. zorros). Dos fechados radiocarbónicos sobre restos de Mylodon darwinii arrojaron una antigüedad de $11590 \pm 100$ AP (PITT-0940) y $13400 \pm 90$ AP (AA-11498) (Borrero et al. 1997). Se suma a éstos, un fechado taxón directo en el incisivo de Vicugna de 13100 \pm 70 AP (Weinstock et al. 2009).

Durante 2008 se retomaron las investigaciones en el sitio en el marco del Proyecto FONDECYT 1070709, excavando las unidades 4D y 4E, y concluyendo la cuadrícula 4C. En esta oportunidad se recuperaron 7.906 especimenes óseos (Labarca 2008). En términos generales, las modificaciones naturales y composición faunística es compatible con los resultados publicados por Borrero et al. (1997) (Labarca 2008). Durante el análisis de los materiales óseos de esta campaña de excavación, se constató la presencia de un importante número de elementos postcraneales de Camelidae con un tamaño compatible con la actual vicuña. Lo anterior, motivó la revisión de los antiguos materiales del yacimiento, confirmando la presencia sistemática de unidades anatómicas de camélidos "pequeños", los que por su tamaño fueron asignadas provisoriamente a Vicugna sp. Para aumentar el conocimiento acerca de la distribución temporal de esta forma, dos elementos esqueletales (segunda y primera falange) de las excavaciones de 2008 fueron datadas por AMS en $13545 \pm 100$ AP (Ua-36261) y $13200 \pm 100$ AP (Ua-36262) respectivamente.

\section{Material y métodos}

Los materiales analizados provienen de las distintas campañas de excavación efectuadas en CLS-4. Debido a la fragmentación del conjunto óseo se seleccionaron falanges y huesos cortos (tarsos y carpos, exceptuando astrágalo y calcáneo). Ambos grupos de elementos anatómicos han sido utilizados en arqueología y paleontología para la identificación de las distintas formas de la familia Camelidae (Cartajena 2002, Izeta 2004, Grant 2008, Kent 1982, Menegaz et al. 1989). En el análisis fueron incluidos sólo aquellos elementos de individuos adultos los que por su tamaño cualitativo fueron adscriptos preliminarmente a Vicugna sp. (Tablas 1 y 2) (Figura 2). Para el caso de las falanges, se consideraron aquellos especimenes que tuvieran sus epífisis proximales fusionadas. Se incluyeron además, fragmentos de falanges distales con sus carillas articulares y bordes bien marcados. Este mismo criterio se aplicó para seleccionar los huesos cortos, ya que tienen un sólo centro de osificación (Cartajena 2002). Las falanges fueron separadas en delanteras y traseras siguiendo la metodología de Kent (1982) y Cartajena (2002), y medidas de acuerdo a los protocolos de von den Driesch (1976), L'Heureux (2008) y Scherer et al. (2007). Los huesos cortos se mensuraron de acuerdo a Cartajena (2007). La totalidad de las medidas fueron obtenidas mediante el uso de un calibre digital de 0,01 mm de precisión y relevadas por un único observador, con el objeto de evitar error interpersonal.

Se realizó un análisis exploratorio uni y multivariado aplicando las siguientes herramientas:

1. Técnicas univariadas: Se utilizó la técnica de diferencias logarítmicas de Meadow (Meadow 1987), la que tiene por objeto comparar las distancias entre medidas teniendo como base un individuo estándar. En este caso se utilizaron como referencia los especimenes paleontológicos de Cueva Lago Sofía 4: CEHA 45742 para las primeras falanges anteriores; CEHA 47469 para las primeras falanges posteriores y CEHA 48349 para la segunda falange posterior (Tabla 1), los que fueron comparados con promedios de medidas de Lama guanicoe de Patagonia, Vicugna vicugna, y con medidas de Vicugna gracilis.

2. Técnicas multivariadas: Se utilizó el coeficiente de simi- 


\begin{tabular}{|c|c|c|c|c|c|c|}
\hline $\mathrm{N}^{\circ}$ registro & Elemento & Later & GD & GB & $\mathrm{GH}$ & $\mathrm{LH}$ \\
\hline CEHA 48530 & Carpo Ulnar & Der & 18,81 & 11,46 & 14,8 & - \\
\hline CEHA 45976 & Carpo Ulnar & $\mathrm{Izq}$ & - & 12,71 & 14,55 & - \\
\hline CEHA 48141 & Carpo Ulnar & Der & 23,29 & 12,85 & 17,64 & - \\
\hline CEHA 48599 & Carpo Ulnar & Der & 20,82 & 11,36 & 15,46 & - \\
\hline CEHA 47324 & Carpo Ulnar & Izq & 20,95 & 11,7 & 16,39 & - \\
\hline CEHA 85959 & Carpo Ulnar & Der & - & 11,15 & 15,05 & - \\
\hline CEHA 86422 & Carpo Ulnar & $\mathrm{Izq}$ & 20,58 & - & 13,93 & - \\
\hline CEHA 86704 & Carpo Ulnar & Izq & 22,43 & 11,35 & 16,19 & - \\
\hline CEHA 46126 & Carpo Radial & $\mathrm{Izq}$ & 20,93 & 12,44 & 15,94 & - \\
\hline CEHA 47845 & Carpo Radial & Der & 21,97 & 13,39 & 15,85 & - \\
\hline CEHA 48062 & Carpo Radial & Der & 22,74 & 14,02 & 16,26 & - \\
\hline CEHA 86188 & Carpo Radial & $\mathrm{Izq}$ & 20,1 & 13,44 & 16,07 & - \\
\hline CEHA 46679 & Carpo Radial & Der & 20,73 & 13,37 & 16,53 & - \\
\hline CEHA 46621 & Carpo Radial & $\mathrm{Izq}$ & 20,97 & 14,52 & 15,19 & - \\
\hline CEHA 48062 & Segundo carpiano & Izq & 11,06 & 9,13 & 11,15 & - \\
\hline CEHA 86120 & Segundo carpiano & Der & 12,24 & 9,51 & 12,06 & - \\
\hline CEHA 86036 & Tercer carpiano & $\mathrm{Izq}$ & 17,26 & 14,42 & 9,02 & - \\
\hline CEHA 48142 & Tercer carpiano & Der & 18,51 & 14,47 & 9,95 & - \\
\hline CEHA 86075 & Cuarto carpiano & Izq & 23,15 & 14,75 & 9,08 & - \\
\hline CEHA 86073 & Cuarto carpiano & $\mathrm{Izq}$ & 25,44 & 12,22 & - & - \\
\hline CEHA 48529 & Cuarto carpiano & Der & 26,87 & 15,89 & 11,33 & - \\
\hline CEHA 47843 & Tarso central & Der & 22,25 & 14,9 & 15,54 & 6,8 \\
\hline CEHA 40061 & Tarso central & Der & 22,49 & 15,54 & 17,53 & 6,8 \\
\hline CEHA 47844 & Tercer tarsiano & Der & 17,54 & 13,26 & 8,75 & - \\
\hline CEHA 47321 & Tercer tarsiano & $\mathrm{Izq}$ & 17,22 & - & 9,07 & - \\
\hline CEHA 48341 & Cuarto tarsiano & Der & 29,76 & 17,62 & - & - \\
\hline CEHA 47842 & Cuarto tarsiano & Der & 29,02 & 18,75 & 19,8 & - \\
\hline CEHA 46619 & Cuarto tarsiano & Der & 28,28 & 18,67 & 19,2 & - \\
\hline CEHA 86180 & Cuarto tarsiano & Der & 26,34 & 17,29 & 15,69 & - \\
\hline CEHA 86119 & Maléolo lateral & Der & 16,08 & 9,06 & 14,55 & - \\
\hline CEHA 86123 & Maléolo lateral & $\mathrm{Izq}$ & 19,56 & 12,29 & 17,37 & - \\
\hline CEHA 46628 & Maléolo lateral & Izq & 20,04 & 11,26 & 19,2 & - \\
\hline
\end{tabular}

Tabla 1. Medidas ( $\mathrm{mm}$ ) de tarsianos y carpianos de CLS-4. Der: derecho; Izq: izquierdo

litud City-Block o Manhattan-Distance, cuyos resultados se volcaron en una matriz de similitud de distancia. A las matrices resultantes se les aplicó un análisis de agrupamiento UPGMA (Unweighted Pair Group Using Arithmetic Averages), resultando un fenograma para cada elemento (Menegaz et al. 1989). En paralelo, se realizó un análisis de componentes principales (ACP) a partir de matrices de correlaciones, con el objeto observar agrupamientos entre los distintos individuos que componen la muestra, utilizando aquellos componentes que explican de mejor manera la varianza del conjunto. Los resultados fueron expresados gráficamente.

Las medidas de las muestras de camélidos actuales (Lama guanicoe n:12 y Vicugna vicugna n:9) fueron tomadas de Cartajena (2002) (Lama guanicoe n:9 y Vicugna vicugna n:9) y de esqueletos de referencia depositados en el Centro de Estudios del Hombre Austral (CEHA) (Punta Arenas) (Lama guanicoe n:3). Las medidas de piezas asignadas en este trabajo a Vicugna gracilis (Tabla 1) fueron obtenidas de especimenes depositados en el Museo de La Plata (MLP) y Museo de Ciencias Naturales Bernardino Rivadavia (MACN), Argentina, todas provenientes del área pampeana. Las medidas de la falange anterior de Agua de la Cueva (Argentina) (AC 106) fueron tomadas de Gil et al. (en preparación). Los restos paleontológicos analizados en este trabajo se encuentran depositados en las colecciones del Centro de Estudios del Hombre Austral, Punta Arenas, Chile (los números de registro se encuentran en las Tablas 1 y 2). Los análisis osteométricos fueron realizados con el programa PAST ver. 1.93 (Hammer et al. 2001).
Abreviaturas de las variables utilizadas: GD: profundidad máxima, GB: ancho máximo; GH: altura máxima; LH: altura mínima; GL: largo máximo; Bp: ancho máximo de la epífisis proximal; BFp: ancho máximo de la carilla articular proximal; Dp: profundidad máxima de la epífisis proximal; DFp: profundidad máxima de la carilla articular proximal; Bd: ancho máximo de la epífisis distal; SD: ancho mínimo de la diáfisis; SDD: profundidad mínima de la diáfisis; GDd: profundidad máxima de la epífisis distal; SDd: profundidad mínima de la epífisis distal; TLC: profundidad máxima del cóndilo lateral distal.

\section{Resultados}

\section{Técnicas univariadas}

Las figuras 3 a, b y c grafican las distancias logarítmicas de las primeras falanges delanteras y traseras y de la segunda falange trasera considerando sólo Gl, Bp, BFp, Dp, DFp, $\mathrm{Bd}$ y SD debido a la ausencia de las medidas restantes en la literatura consultada. En lo que respecta a las primeras falanges anteriores y posteriores, se aprecia que los elementos de CLS-4 CEHA 45742 y CEHA 47469 poseen en general un tamaño menor que los promedios de Lama guanicoe, registrándose las mayores distancias en los anchos proximales (DFp y BFp), mientras que el ancho de la diáfisis (SD) corresponde a la medida más cercana. En ambas primeras falanges, las menores distancias se registran indudablemente con Vicugna vicugna y Vicugna gracilis, aunque las piezas se comportan de manera distinta. La primera falange anterior posee medidas que comparativamente se alejan más de $V$. vicugna, acercándose en cambio a $V$. gracilis. Este elemento es siempre más grande que el de la actual vicuña, siendo particularmente más largo y robusto en su diáfisis. En relación con $V$. gracilis se puede observar que si bien la falange del taxón pampeano es más larga (Gl) que el espécimen CEHA 45742, éste último presenta anchos epifisiales (Bp, BFp, Bd) similares. Las medidas que disponemos de Agua de la Cueva, en donde se ha identificado una forma más pequeña que el guanaco actual (García et al. 2008), indican que tres de éstas son compatibles con $V$. vicugna, a excepción de $\mathrm{Bp}$, la que aparece mucho más grande que la falange de CLS-4 CEHA 45742 y que V. gracilis (Figura 3a). Esta situación podría deberse a una importante variabilidad individual del elemento de Agua de la Cueva, o bien, a un problema en la toma de las medidas.

Por su parte, la primera falange posterior ocupa una posición intermedia entre ambos taxones pequeños, siendo un poco más grande que la actual vicuña, pero comparativamente más pequeña que los cuatro elementos de V. gracilis. Los anchos y profundidades proximales (Bp, BFp y DFp) son las medidas más cercanas con $V$. vicugna, mientras que el ancho de la diáfisis (SD) es la más lejana. Respecto de las medidas de $V$. gracilis, las cuatro piezas de referencia presentan una distancia considerable con el espécimen CEHA 47469 en términos de su longitud total; 


\begin{tabular}{ccccccccccccc}
\hline$N^{\circ}$ Reg & Elemento & GL & Bp & BFp & Dp & DFp & Bd & SD & SDD & GDd & SDd & TLC \\
\hline CEHA 45742 & Falange 1 Ant & 66,59 & 16,74 & 15,97 & 17 & 16,42 & 13,87 & 10,63 & 9,37 & 12,47 & - & - \\
CEHA 48534 & Falange 1 Ant & 64,87 & 16,88 & - & 16,42 & - & 14,61 & 10,64 & 8,81 & - & 10,87 & - \\
MLP 9-231 & Falange 1 Ant & 71,46 & 15,79 & 15,77 & 17,1 & 16,52 & 13,63 & 10,69 & 11,57 & 14,08 & 11,84 & 12,52 \\
AC 106 & Falange 1 Ant & 60,82 & 20,12 & - & 16,36 & - & 13,2 & - & - & - & - & - \\
CEHA 47469 & Falange 1 Post & 51,37 & 15,53 & 15,3 & 15,56 & 13,85 & 14,34 & 9,91 & 8,36 & 13,14 & 11,01 & 12,84 \\
CEHA 48503 & Falange 1 Post & 56,6 & 17,87 & - & 15,69 & 14,92 & 14,64 & 10,45 & 8,75 & 12,81 & 11,85 & 12,61 \\
CEHA 86664 & Falange 1 Post & - & - & - & - & - & 13,99 & - & - & 13,27 & - & 12,64 \\
MLP 9-228 & Falange 1 Post & 60,41 & 18,34 & 17,53 & 18,55 & 17,43 & 15,83 & 10,68 & 13,59 & 12,83 & 12,61 & - \\
MLP 9-275 & Falange 1 Post & 58,52 & 16,37 & 15,93 & 15,89 & 14,3 & - & 9,62 & 9,14 & - & - & - \\
MLP 9-276 & Falange 1 Post & 76,87 & 19,99 & 19,95 & 20,6 & 19,14 & 16,92 & 11,74 & 15,81 & 13,33 & 13,12 & - \\
MACN 2005 & Falange 1 Post & 61,42 & 16,56 & 16,43 & 16,35 & - & 15,15 & 11,28 & 10 & 13,59 & 12,16 & 13,41 \\
MACN 6980 & Falange 1 Post & 58,14 & 18,73 & 17,97 & 19,23 & 17,54 & 15,36 & 10,78 & 9,16 & 13,49 & 11,45 & 12,66 \\
CEHA 47859 & Falange 2 Ant & - & 14,47 & 13,71 & - & 11,25 & - & - & - & - & - \\
CEHA 86479 & Falange 2 Ant & 32,91 & 14,08 & 13,7 & 11,56 & 10,71 & 13,46 & 11,39 & 9,64 & 13,18 & - \\
CEHA 48349 & Falange 2 Post & 29,9 & 15,22 & 13,98 & 12,17 & 11,74 & 12,64 & 11,58 & 8,57 & 10,41 & - \\
CEHA 46007 & Falange 2 Post & - & 15,63 & 14,05 & 14,59 & 12,15 & - & - & - & - & - \\
CEHA SD & Falange 2 Post & - & 14,39 & 14,87 & 13,53 & 11,66 & - & - & - & - & - \\
CEHA 46717 & Falange 2 Post & - & 15,98 & 15,13 & 14,38 & 13,75 & - & - & - & - & - \\
\hline
\end{tabular}

Tabla 2. Medidas $(\mathrm{mm})$ de primeras y segundas falanges de CLS-4 y de Vicugna gracilis utilizadas como referencia. Ant: Anterior; Post: Posterior.

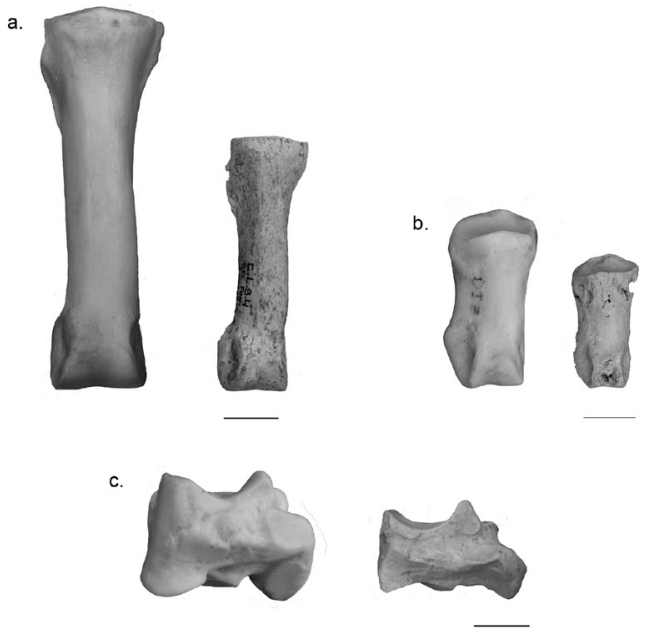

Figura 2. Restos óseos de Vicugna de CLS-4 (derecha), comparados con material actual de Lama guanicoe (derecha). a. Primera falange posterior; b. segunda falange anterior; c. Cuarto tarsiano. La barra mide $1 \mathrm{~cm}$.

el resto de las medidas se agrupan en dos subconjuntos, dando cuenta posiblemente de variabilidad intraespecífica de este taxón (Figura 3b). Se destaca las distancias en el ancho diafisiario (SD) de tres de las cuatro piezas, indicando que los restos de $V$. gracilis serían aún más robustos ("intermedios") que el espécimen CEHA 47469.

La segunda falange posterior de CLS-4 (CEHA 48349) presenta igualmente una posición intermedia entre $V$. vicugna y L. guanicoe, aunque las distancias son ostensiblemente menores con el primero de éstos taxones. La mayor cercanía con la actual vicuña se registra con el largo máximo, mientras que el ancho de la diáfisis corresponde a la mayor distancia, situación también observada en las primeras falanges (Figura 3c). La ausencia de segundas falanges asignadas a $V$. gracilis impide comparaciones con esta especie extinta. Aunque se trata de un número muy pequeño de muestras, la mayor robustez diafisiaria en las falanges de la vicuña de Última Esperanza en relación con la vicuña actual merece ser considerada para futuros trabajos con un mayor número de muestras.

\section{Técnicas multivariadas}

Del total de elementos analizados, en este trabajo se presentan sólo los resultados para las falanges (primeras y segundas), considerando las variables Gl, Bp, BFp, Dp, DFp, Bd y SD; carpo ulnar, carpo radial y cuarto tarsiano, considerando las variables GD, GD y GH, debido fundamentalmente a que se trata de las unidades óseas más representativas y/o con mayor número de elementos identificados.

En lo que respecta a la primera falange anterior, el análisis de coeficientes de similitud permite separar con claridad un subgrupo conformado por los elementos de referencia de Lama guanicoe y otro que se agrupa a Vicugna vicugna, V. gracilis y la pieza CEHA 45742, aún cuando éstos dos últimos generan un conjunto separado (Figura 4a). Por su parte, el gráfico generado a partir ACP permite observar que el componente 1 (que explica un 95,3\% de la varianza, Anexo 2, Tabla 1) segrega en términos generales dos subgrupos principales, compuestos por los elementos de referencia de $L$. guanicoe y $V$. vicugna + V. gracilis + CEHA 45742 respectivamente. En este último subgrupo, V. gracilis + CEHA 45742 se separan levemente de $V$. vicugna principalmente en el segundo componente, el que sin embargo explica el 2,5\% de la varianza (Anexo 2, Tabla 1). La falange de Última Esperanza es, no obstante, ligeramente más pequeña que su contraparte pampeana (Figura 5a).

El fenograma generado para la primera falange posterior separa a los especímenes en tres grandes grupos: Lama guanicoe + Vicugna gracilis (aunque éste último confor- 

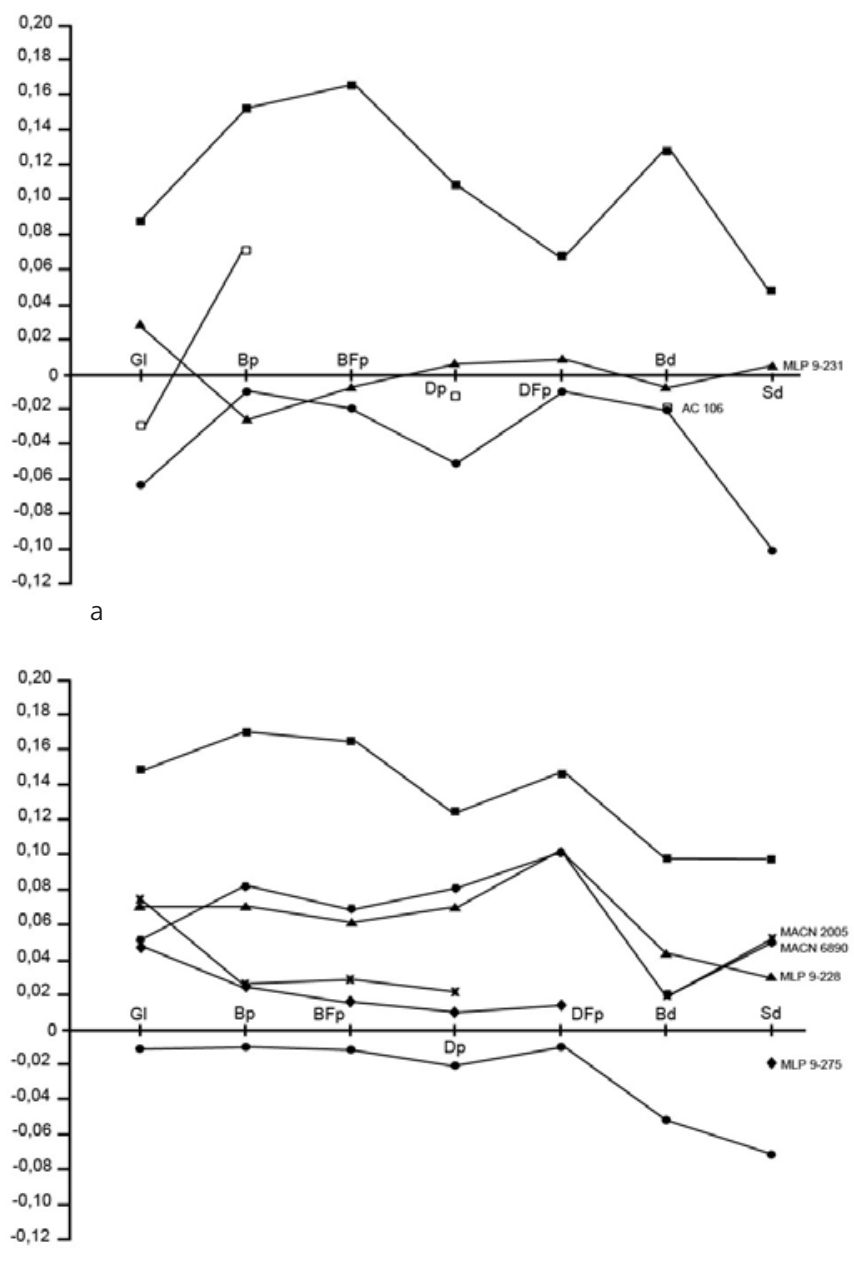

b

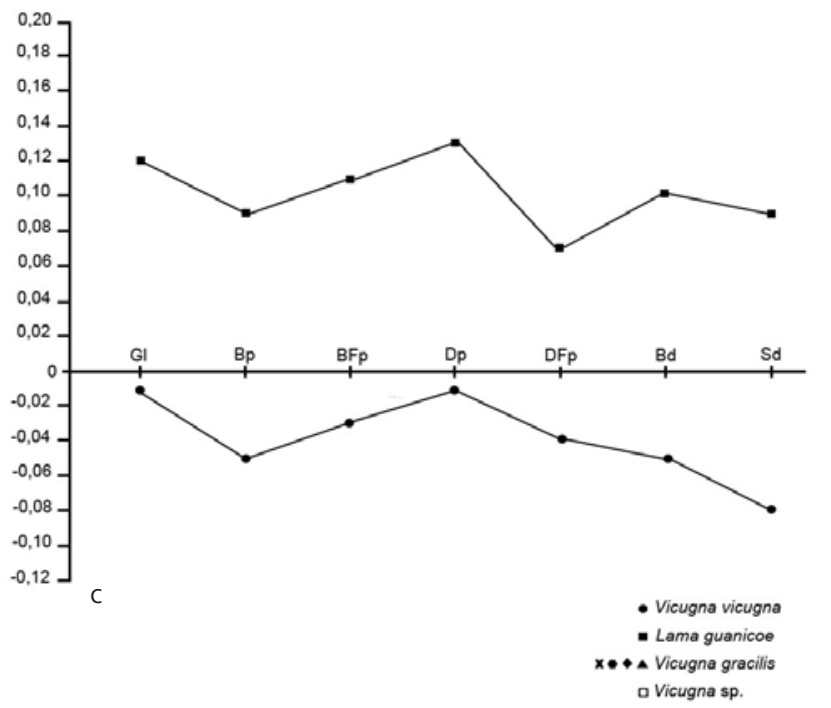

Figura 3. a. Distancias logarítmicas de falanges de la familia Camelidae, utilizando como estándar los elementos paleontológicos de CLS-4. a. Primera falange anterior; b. Primera falange posterior; c. Segunda falange posterior.

mado a su vez un subgrupo), Vicugna vicugna + CEHA 47469 y, finalmente, un conjunto de guanacos de gran tamaño, los que corresponden a aquellos de referencia depositados en el CEHA (Figura 4b). En principio, estos resultados sustentarían una eventual diferenciación de V. gracilis como taxón independiente de $V$. vicugna, el que métricamente se encontraría más relacionado con los guanacos actuales de menor tamaño utilizados como referencia, lo que confirmaría el tamaño "intermedio" sugerido por Menegaz et al. (1989). La pieza CEHA 47469, por su parte, debería ser asignada a $V$. vicugna, ya que se encuentra dentro del rango de variabilidad métrica actual de esta especie. El ACP entrega, en términos generales, resultados similares a los registrados en el análisis de coeficientes de similitud. En este caso, el componente 1 (que explica el 96,6\% de la varianza, Anexo 2, Tabla 2), permite separar a los individuos en cuatro grandes subgrupos: $L$. guanicoe, L. guanicoe "grandes", V. gracilis y V. vicugna. El espécimen CEHA 47469 cae dentro de la variabilidad de la actual vicuña. Esta situación probablemente se deba a que el largo máximo corresponde a la variable que más contribuye en este componente (Anexo 1, Tabla 2). Por último, es interesante destacar la presencia de una pieza asignada a $V$. gracilis de tamaño compatible con L. guanicoe (MLP 9-276), la que probablemente se encuentra mal determinada (Figura 5b).

En lo que respecta a la segunda falange posterior, tanto el fenograma obtenido a partir del análisis de distancias como el análisis de componentes principales, permiten incluir el espécimen CEHA 48349 dentro de Vicugna vicugna (Figuras 4c y 5c). Se aprecia en el ACP que la falange CEHA 48349 se aleja de los elementos de V. Vicugna de referencia en el segundo componente, situación que se explicaría por variaciones en la forma de los elementos. Este componente, sin embargo, explica sólo un 2,2\% de la varianza (Anexo 2, Tabla 3)

Los resultados obtenidos con los huesos cortos son, en términos generales, coherentes con lo observado para la primera falange posterior y la segunda falange posterior. El fenograma generado para el carpo radial permite separar con claridad a Lama guanicoe y Vicugna vicugna + CLS-4. Sin embargo, dentro de éste último es posible advertir dos agrupaciones, una conformada principalmente por los elementos del sitio pleistocénico más tres casos de V. vicugna "grandes", y otra compuesta exclusivamente por V. vicugna (Figura 4d). En menor medida, esto último también se aprecia en el gráfico de componentes principales, en donde sobre el primer componente se observa que cinco especímenes de CLS-4 (CEHA 48602, 46621, 46679,47845 y 86188 ) presentan tamaños mayores que los huesos de $V$. vicugna de referencia, aunque lejos de los elementos de L. guanicoe (Figura $5 d$ ). Por su parte, los resultados de los análisis de coeficientes de similitud efectuados con el carpo ulnar permiten separar un subgrupo conformado por L. guanicoe y otro compuesto por $V$. vicugna, ubicándose en este último todos los elementos del yacimiento de CLS-4 (Figura 4e). Resultados homologables se observan en el ACP, en donde el primer componente (que explica el 96,45\% de la varianza, Anexo 2, Tabla 5) separa a L. guanicoe de V. Vicugna + CLS-4. Dentro del primer grupo se aprecia a su vez, una relativa homogeneidad en los tamaños y una mayor variabilidad 

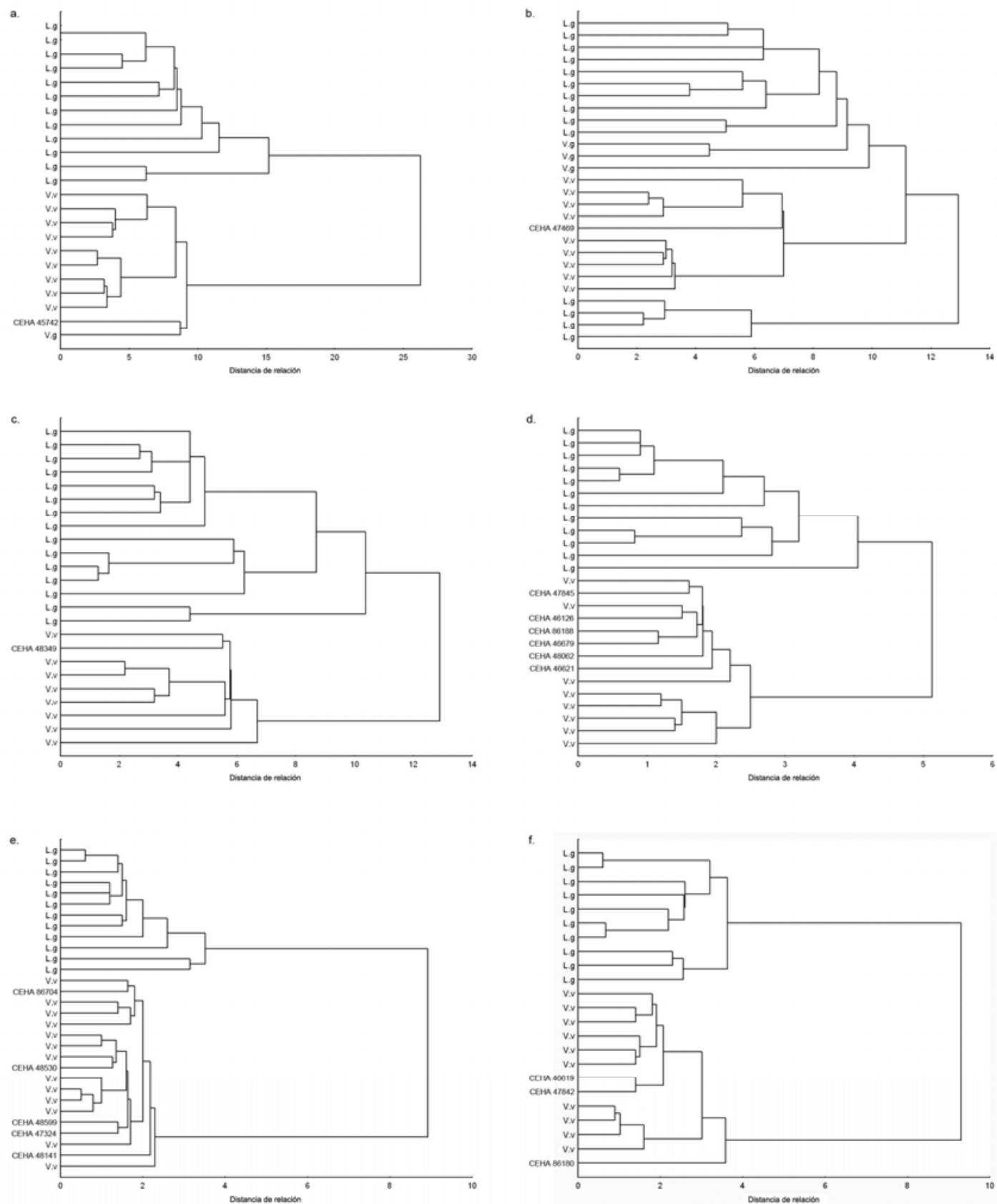

Figura 4. Fenogramas (UPGMA) de relaciones de similitud. a. Primera falange anterior b. Primera falange posterior; c. Segunda falange posterior, d. Carpo radial, e. Carpo Ulnar, f. Cuarto tarsiano. L.g: Lama guanicoe; V.v: Vicugna vicugna; V.g: Vicugna gracilis.

en las formas. La excepción la conforman dos grandes elementos de L. guanicoe, los que corresponden a aquellos de referencia depositados en el CEHA. Una situación similar se aprecia con los carpos ulnares de CLS-4, los que presentan resultados positivos en el segundo componente (Figura 5e). Por último, respecto de los resultados obtenidos para el cuarto tarsiano, el fenograma conformado a partir del análisis de coeficientes de similitud separa los subgrupos $L$. guanicoe y $V$. vicugna. Los tarsianos de CLS4 (CEHA 46619, 47862, 86189) se asocian en todos los casos al grupo "pequeño". El ACP refuerza esta impresión, ya que los especimenes de CLS-4 se correlacionan con los elementos de $V$. vicugna utilizados como referencia en el primer componente (Figura $4 f$ y $5 f$ ).

\section{Discusión}

\section{Taxonomía}

Menegaz et al. (1989) utilizaron principalmente criterios morfológicos ubicados en los incisivos para la segregación de las dos especies adscritas en este trabajo al género Vicugna. De acuerdo a éstos autores, el mayor o menor grado de imbricación en los incisivos, el mayor o menor desarrollo en las facetas de desgaste en bisel y la presencia o ausencia de esmalte en la cara labial de éstos serían los principales indicadores diagnósticos para diferenciar entre $V$. vicugna y $V$. gracilis. Paralelamente, los autores realizaron análisis osteométricos multivariados con el 

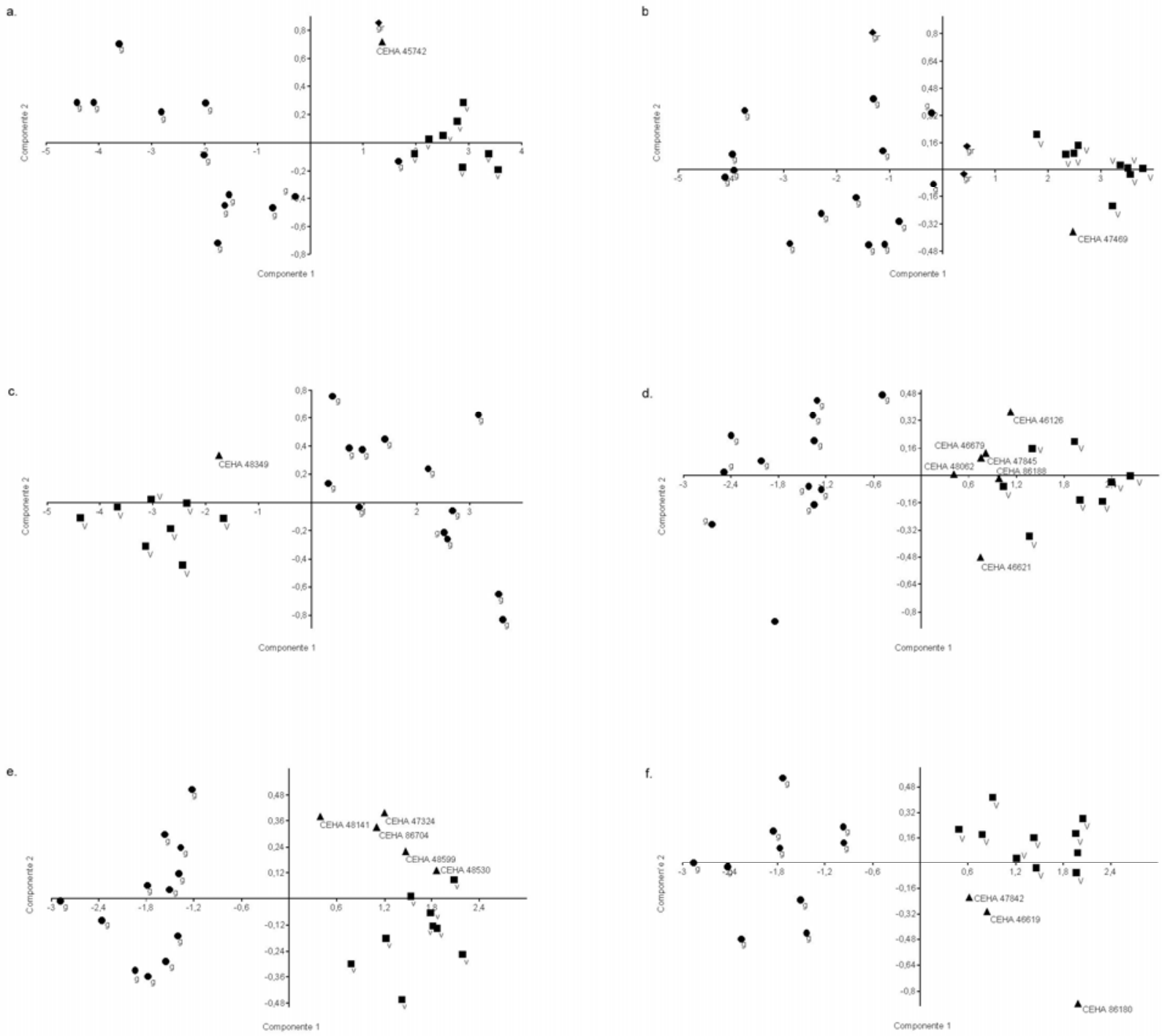

Figura 5. Gráficos de componentes principales a. Primera falange anterior; b. Primera falange posterior; c. Segunda falange posterior, d. Carpo radial, e. Carpo ulnar, f. Cuarto tarsiano. g: Lama guanicoe; v: Vicugna vicugna; gr: Vicugna gracilis. Ver texto para explicación.

objeto de aportar mayor evidencia a la diagnosis de ambas formas, concluyendo que no todas las unidades anatómicas arrojaban diferencias suficientes como para distinguir especies. En efecto, los metapodios fueron los únicos elementos métricamente distinguibles; las falanges sólo permitían diferencias entre Lama guanicoe y Vicugna, mientras que los astrágalos no permitían ni siquiera una diferenciación genérica (Menegaz et al. 1989).

Pese a las aprensiones indicadas por Menegaz et al. (1989), en este trabajo se consideraron falanges y huesos cortos para discutir la presencia del género Vicugna en Cueva Lago Sofía 4 y explorar la posibilidad de realizar asignaciones específicas con el material disponible. En términos generales, los resultados de los análisis uni y multivariados efectuados a estas unidades separan el grupo de camélidos conformados por los representantes patagónicos de L. guanicoe del grupo conformado por el género Vicugna ( $V$. vicugna $+V$. gracilis), a excepción del fenograma de la primera falange posterior, en donde el grupo L. guanicoe se separó en dos subconjuntos (Figura 4b). La totalidad de los especímenes de CLS-4 (primera falange anterior y posterior, segunda falange posterior, carpo ulnar, carpo radial y cuarto tarsiano) se agruparon dentro del subconjunto Vicugna lo que en principio confirma la asignación taxonómica preliminar basada en impresiones cualitativas de tamaño. Los análisis univariados y multivariados en donde se incluyeron materiales de comparación de $V$. gracilis y $V$. vicugna (falange 1 anterior y posterior), indicaron que las falanges primeras de CLS-4 (CEHA 45742 y CEHA 47469) presentaron un tamaño menor o similar que los elementos anatómicos de $V$. gracilis, y mayor o similar que las unidades anatómicas de $V$. vicugna utilizadas como referencia. Respecto de los huesos cortos, si bien no se contó con restos de $V$. gracilis comparativos, el análisis de agrupamientos UPGMA indicó que todos los huesos cortos incluidos en el presente estudio se reúnen con aquellos de $V$. vicugna sin generar subgrupos independientes. Resultados similares arrojaron los análisis de componentes principales, ya que el primer componente permitió la inclusión de los materiales en estudio dentro de la variabilidad observa- 
da para $V$. vicugna, a excepción de lo registrado en tres carpos radiales, en donde se detectaron especimenes de tamaños levemente mayores a los observados para este taxón (Figura 5d).

Diversos investigadores han reparado en la gran variabilidad en el tamaño de Lama guanicoe tanto en términos geográficos como cronológicos. En efecto, L'Heureux (2008) ha documentado una disminución progresiva en el tamaño de $L$. guanicoe desde el Pleistoceno al Holoceno en Patagonia, mientras que Mengoni-Goñalons y Yacobaccio (2006) han demostrado importantes variaciones métricas entre los guanacos andinos y surpatagónicos, debido a que el tamaño corporal de este taxón está correlacionado negativamente con la latitud ("Ley de Bergmann"). Esta característica es también propia de Vicugna vicugna, ya que la subespecie sureña ( $V$. v. vicugna) es comparativamente más grande que la norteña (V. v. mensalis) (Wheeler 1995, 2006). Tendiendo en cuenta los resultados de los análisis uni y multivariados y considerando la variaciones en la familia Camelidae de acuerdo a la Ley de Bergmann, los elementos en estudio son asignados específicamente a Vicugna vicugna. Como se mencionó, los elementos anatómicos utilizados en el presente trabajo arrojaron tamaños similares o superiores a la vicuña actual, diferencias que a nuestro juicio son explicables por la procedencia latitudinal de las muestras. Esta asignación se sustenta igualmente en el hecho de que las falanges primeras de CLS-4 no superaron en tamaño a los elementos de $V$. gracilis comparativos de la región pampeana. Lo anterior confirma las descripciones de los materiales dentales realizadas por Prieto y Canto (1997) y los estudios de ADN antiguo (Weinstock et al. 2009).

En el presente trabajo se incluyó de manera exploratoria una primera falange proveniente del sitio arqueológico Agua de la Cueva (AC 106), ubicado en la cordillera Andina de la región de Cuyo ( $\left.32^{\circ} 50^{\prime} S\right)$. Ésta pieza presentó un tamaño intermedio entre $V$. vicugna de referencia y la pieza CEHA 45742 (a excepción de Bp), sugiriendo que el taxón registrado en la precordillera de Mendoza podría corresponder a Vicugna vicugna. Este dato necesita ser contrastado con nuevos análisis, ya que su localización intermedia entre el área surpatagónica y puneña resulta importante para discutir posibles rutas de dispersión de este taxón a través de una ruta andina.

Weinstock et al. (2009) recientemente han criticado las asignaciones de $V$. gracilis del área patagónica y pampeana argentina centradas exclusivamente en un mayor tamaño en relación a la actual vicuña. La base de su argumento radica en que, producto de variaciones latitudinales, los especimenes en cuestión podrían corresponder simplemente a ejemplares de $V$. vicugna de mayor tamaño. Los resultados de los análisis osteométricos uni y multivariados efectuados en este trabajo, sustentarían en principio una diferenciación entre $V$. gracilis del Pleistoceno de la región Pampeana y $V$. vicugna del Pleistoce- no final de Última Esperanza, ya que aún considerando eventuales variaciones latitudinales, las falanges primeras anteriores y posteriores de $V$. gracilis demostraron ser mayores o iguales que los elementos provenientes de Cueva Lago Sofía 4 y fueron siempre más grandes que las falanges de $V$. vicugna actuales. La falta de elementos anatómicos de camélidos pequeños provenientes de sitios arqueológicos del área Patagónica argentina (p.e Los Toldos, La María, Piedra Museo, Menegaz et al. 1989; Miotti y Salemne 1999; Paunero et al. 2004) no permite, por el momento, contrastar osteométricamente la hipótesis de Weinstock et al. (2009), aunque el tamaño registrado en la primera falange de Agua de la Cueva podría aportar luces al respecto.

\section{Consideraciones paleoecológicas y biogeográficas}

En la actualidad, el género Vicugna posee una distribución geográfica muy acotada, la que se relaciona fundamentalmente con sectores altoandinos por sobre los 3.000 msnm (Franklin 1982, González et al. 2000. Wheeler 1995, 2006). Menegaz et al. (1989) han argumentado que la adaptación de este género no se relaciona con las condiciones de altura, sino más bien con la vegetación que allí se desarrolla, compuesta principalmente por gramíneas duras y rígidas con un alto contenido silíceo. En efecto, a diferencia del guanaco, la vicuña es una animal fundamentalmente pastador, con un aparato bucal especializado para el procesamiento de vegetales resistentes y abrasivos propios de climas agrestes, lo que se evidencia en la presencia de incisivos de crecimiento continuo, con facetas de desgaste en bisel (generadas por la presencia de esmalte sólo en la cara lingual) y escasa o nula imbricación entre éstos (Menegaz et al. 1989). La presencia de representantes fósiles del género Vicugna fuera del área andina se explicaría por la distribución más amplia de las gramíneas duras, las que durante el Pleistoceno se habrían repartido mas extensivamente por el área pampeana y patagónica, debido a las condiciones climáticas frías imperantes en el área (Menegaz et al. 1989). Esta hipótesis permitiría explicar la presencia de V. vicugna en el Pleistoceno final (ca. 13500 AP - 10300 AP) de Patagonia meridional, ya que estudios paleoclimáticos llevados acabo en el área de Última Esperanza y en el área altoandina norchilena y sur Boliviana han demostrado condiciones ambientales relativamente similares, concordancia conocida en la actualidad como "Diagonal Árida" (Abraham et al. 2000). Cárdenas (2006) y Villa-Martínez y Moreno (2007) coinciden en señalar para el Pleistoceno final del área adyacente a Lago Sofía 4, la existencia de paisajes abiertos con un predominio de taxa de pastizales (principalmente gramíneas) y en menor medida arbustivas, asociadas a un clima frío y comparativamente más húmedo que el actual. Similares condiciones han sido inferidas en el lago Titicaca (Sylvestre et al. 1999), laguna Miscanti, Chile (Grosjean et al. 2001) y la cuenca del Salar de Atacama (Betancourt et al. 2000, Latorre et al. 2003), entre otros. Particularmente, en esta 
última área se ha definido un período comprendido entre los 16200 y 10500 AP dominado por condiciones frías y comparativamente más húmedas con presencia de un ambiente de estepa hasta por lo menos los 1000 msnm (Betancourt et al. 2000, Latorre et al. 2003).

Los registros de V. vicugna desaparecen de Patagonia meridional chilena en torno a los 10.600 AP (Massone 2004); sin embargo en el área puneña este taxón persiste hasta la actualidad (Wheeler 2006). Esta situación posiblemente se deba a que las condiciones ambientales compartidas entre ambos sectores comienzan a variar en ciertos aspectos claves durante la transición Pleistoceno-Holoceno. En el área adyacente a la Cueva Lago Sofía 4, los estudios de polen indican un abrupto aumento en la representación de taxa boscosas (Nothofagus) asociado a la disminución de gramíneas y un aumento en la temperatura en un momento cercano a los 10.340 AP. Este cambio se vuelve más significativo en torno a los 9.980 AP, lo que marcaría el inicio del Holoceno (Cárdenas 2006). En el área puneña en cambio, el inicio del Holoceno se encuentra definido por el descenso de los niveles lacustres asociado a una disminución de las lluvias, aumento de la temperatura y sólo una retracción de las especies vegetales hacia sectores comparativamente más elevados (Grosjean et al. 2001, Latorre et al. 2003). La desaparición de Vicugna vicugna exclusivamente en el área Patagónica se explicaría, en parte, por el aumento del bosque de Nothofagus detectado hacia finales del Pleistoceno debido a la adaptación de este taxón a ambientes abiertos (p.e dieta pastadora) (Cionne et al. 2009, Menegaz et al. 1989), situación que posiblemente se vio agravada por su marcada territorialidad y nulas conductas migratorias (Franklin 1982, González et al. 2000, Wheeler 1995). En este escenario, $\checkmark$. vicugna se habría visto desfavorecida en una eventual competencia con otros taxones de dietas más generalizadas y hábitos más flexibles como el guanaco (González et al. 2006). Se debe considerar además, que el registro faunístico del yacimiento Cueva Lago Sofía 4 habría sido generado por un gran felino extinto, por lo que no se puede descartar que una depredación sistemática de Vicugna vicugna por parte de grandes carnívoros pudo haber contribuido a la merma de pequeñas poblaciones patagónicas de este Camelidae. A esto se debe sumar la presencia, aunque escasa, de este taxón en algunos yacimientos arqueológicos finipleistocénicos como Tres Arroyos 1 (Massone 2004), lo que indica su consumo por los primeros grupos de humanos que habitaron en el área. En suma, la conjunción de factores medioambientales, adaptativos, etológicos y culturales habrían contribuido paulatinamente a la retracción de su antigua distribución geográfica hasta configurar su dispersión actual, asociada exclusivamente a ambientes altoandinos con una mayor estabilidad climática.

\section{Conclusiones}

Este trabajo ha constatado, a partir de un análisis osteométrico uni y multivariado, la presencia de Vicugna vicugna en el Pleistoceno final de Última Esperanza, complementando con una nueva línea de evidencia las conclusiones arribadas por los análisis morfológicos y paleogenéticos (Prieto y Canto 1997, Weinstock et al. 2009). El registro de este taxón se explicaría fundamentalmente por la presencia de pastos duros y climas fríos, los que se habrían desarrollado tanto en el área patagónica como puneña ("Diagonal Árida"). En este sentido, la distribución actual de Vicugna vicugna es sin duda un remanente de una extensión mucho mayor, la que alcanzó su máxima expresión hacia el Último Máximo Glacial.

La presencia de este nuevo integrante del elenco paleofaunístico en Cueva Lago Sofía 4 y Tres Arroyos 1, abre la posibilidad de detectar esta forma en otros sitios arqueológicos/paleontológicos contemporáneos del área (e.g. Cueva del Milodón, Saxon 1976; Cueva del Medio, Nami y Menegaz 1991), los que fueron estudiados sin considerar esta posibilidad. Futuros trabajos en esta línea permitirán aportar nuevos datos respecto del rol de las vicuñas en las estrategias de subsistencia de los primeros grupos que habitaron el sector, su relación con los grandes carnívoros extintos y el papel que ambos habrían jugado en su desaparición de Patagonia, entre otros.

\section{Santiago de Chile y Puerto Natales, julio de 2009}

\section{Agradecimientos}

Agradecemos a Lucas Pomi y Marcelo Regueiro (División paleontología de Vertebrados del Museo de La Plata) y a Alejandro Kramarz (División Paleontológica del Museo de Ciencias Naturales Bernardino Rivadavia) por las facilidades para revisar las colecciones paleontológicas allí depositadas. A G. Neme, A. Gil y A. García por facilitar las medidas de la falange de Agua de la Cueva. B. González, E. Calás y D. Rubilar (Museo Nacional de Historia Natural) revisaron una primera versión del manuscrito. J.F. Blanco tradujo el resumen al inglés. C. Scherer (Seção de Paleontologia Museu de Ciências Naturais Fundação Zoobotânica do Rio Grande do Sul) y un revisor anónimo colaboraron de manera significativa a mejorar este manuscrito. Finalmente, agradecemos a A. D. Izeta por su invitación a participar en este número de RMA. Este trabajo fue financiado por el Proyecto Fondecyt 1070709.

\section{Bibliografía}

Abraham, E.M., K. Garleff, H. Liebricht, A.C. Regairaz, F. Schäbitz, F.A. Squeo, H. Stingl, H. Veit y C. Villagrán 2000. Geomorphology and paleoecology of the arid diagonal in Southern South America. Zeitschrift für Angewandte Geologie: Sonderheft SH 1: 55-61.

Betancourt, J.L., C. Latorre, J.A. Rech, J. Quade y K.A. Rylander 2000. A 22,000-year record of monsoonal precipitation from northern Chile's Atacama Desert. Science 289: 1542-1546.

Borrero, L.A., F. M. Martín y A. Prieto 1997. La Cueva Lago 
Sofía 4, Última Esperanza: una madriguera de felino del Pleistoceno tardío. Anales del Instituto de la Patagonia, Serie Ciencias Humanas 25:103-122.

Boule, M. y A. Thevenin 1920. Mammiféres fossiles de Tarija. Misión Scientifique, Crequi. Montfort et E. Senechal de la Grange, Tomo VII. Paris (Soudier).

Cabrera A. 1932. Sobre los camélidos fósiles y actuales de la América Austral. Revista del Museo de la Plata 33:89-117.

Cabrera A. 1934. Los yaguares vivientes y extinguidos de la América austral. Notas Preliminares del Museo de la Plata 2:34-50.

Cárdenas, M. 2006. Vegetación y clima postglacial en Última Esperanza, Patagonia sur de Chile. Facultad de Ciencias, Universidad de Chile, Chile. 78 p. Universidad de Chile.

Cartajena, M.I. 2002. Los conjuntos arqueofaunísticos del Arcaico Temprano en la Puna de Atacama, Norte de Chile. Área de Historia y Cultura, Universidad de Berlín, Alemania, 189 pp. ABESY Vertiebs GMBH.

Cartajena, M.I. 2007. Una propuesta metodológica para la estandarización de medidas de huesos cortos de camélidos y para la determinación de sexo. Werkén 10:49-62.

Cione A.L., E.P. Tonni y L.H. Soibelzon 2009. Did Humans Cause the Late Pleistocene-Early Holocene Mammalian Extinctions in South America in a Context of Shrinking Open Areas? Haynes, G., editor, American Megafaunal Extinctions at the End of the Pleistocene, 125-144. Springer Science + Business Media B.V, New York.

Franklin, W. 1982. Biology, ecology and relationship to man of the South American camelids. Mares, M.A., y $\mathrm{H}$. H. Genoways, Mamalian biology in South America, Pymatuning Symposia in Ecology 6, pp. 457-489. University of Pittsburgh, Linesville, Pennsylvania.

García, A., E. Martínez Carretero y M.A. Dacar 2008. Presence of Hippidion at two sites of western Argentina. Diet composition and contribution to the study of the extinction of Pleistocene megafauna. Quaternary International 180:22-29.

Gervais, H. y F. Ameghino 1880. Los mamíferos fósiles de la América del Sur. Sabih e Igon, París y Buenos Aires.

Gil, A.F., G.A. Neme y A. García. Explotación faunística en los Andes Meridionales durante el Pleistoceno Final y los inicios del Holoceno: el Registro de Agua de La Cueva (Mendoza, Argentina). En preparación.

González, B.A., R.E. Palma, B. Zapata y J.C. Marín 2006.
Taxonomic and biogeographic status of guanaco Lama guanicoe (Artiodactyla, Camelidae). Mammal Review 36:157-178.

González, G., J.C. Torres-Murra y A. Muñoz-Pedreros 2000. Orden Artiodactyla. Muñoz-Pedreros, A. y J. Yánez, editores, Mamíferos de Chile, pp. 189-206, CEA Ediciones, Valdivia.

Grant, J. 2008. El recurso Camelidae en sitios de la Puna Meridional Argentina: Una aproximación osteométrica. Facultad de Filosofía y Letras, Universidad de Buenos Aires, Argentina, 147 pp. Universidad de Buenos Aires.

Gray, J.E. 1837. Description of some new or little known Mammalia, principally in the British Museum collection. Magazine of Natural History 1:577-587.

Grosjean M., J.F.N. van Leeuwen, W.O. van der Knaap, M.A. Geyh, B. Ammann, W. Tanner, B. Messerli, L. Núñez, B. Valero-Garcés y H. Veit. 2001. A 22,000 ${ }^{14} \mathrm{C}$ year BP sediment and pollen record of climate change from Laguna Miscanti $\left(23^{\circ} \mathrm{S}\right)$, northern Chile. Global and Planetary Change 28:35-51

Guerin, C. y M. Faure 1999. Palaeolama (Hemiauchenia) niedae nov. sp., Noveau camelidae du nordeste Brésilien et sa place parmi les Lamini d'Amerique du Sud. Geobios 32:629-659.

Hammer, Ø., D.A.T. Harper y P. D. Ryan 2001. PAST: Paleontological Statistics Software Package for Education and Data Analysis. Palaeontologia Electronica 4(1):1-9.

Hoffstetter R. 1952. Les mammifères Pléistocènes de la République de l' Equateur. Mémoires Société Géologique de France 31(66):1-391.

Izeta, A.D. 2004. Zooarqueología del Sur de los Valles Calchaquíes: Estudio de conjuntos faunísticos del Período Formativo. Facultad de Ciencias Naturales y Museo, Universidad Nacional de la Plata, Argentina, 430 pp.

Kadwell, M., M. Fernández, H. Stanley, R. Baldi, J.C. Wheeler, R. Rosadio y W. Bruford 2001. Genetic analysis reveals the wild ancestors of the llama and the alpaca. Proceedings of Royal Society of London, Biological Series 268:2575-2584.

Kent, J. D.1982. The Domestication and exploitation of the South American camelids: methods of analysis and their application to circum-lacustrine archaeological sites in Bolivia and Peru. Departament of Anthropology, Washington University. St. Louis, Missouri, USA. University Microfilms International Ann Harbor, Michigan.

L'Heureux, G.L. 2008. El estudio arqueológico del proceso coevolutivo entre las poblaciones humanas $y$ 
las poblaciones de guanaco en Patagonia meridional y norte de Tierra del Fuego. BAR International Series 1751. Oxford.

Labarca, R. 2008. Informe de excavaciones paleontológicas del sitio Cueva Lago Sofía 4 (Última Esperanza, Chile). Informe segundo año proyecto FONDECYT 1070709. 36 pp. Manuscrito.

Labarca, R. Los mamíferos del Plio-Pleistoceno de Chile. Rubilar, D. y M. Salaberry, editores, Vertebrados fósiles de Chile. Editorial Universitaria, Santiago. En preparación.

Labarca, R., A. Prieto y V. Sierpe 2008. Sobre la presencia de Smilodon populator Lund (Felidae Machairodontinae) el Pleistoceno tardío de Patagonia meridional. Rubilar, D., A. Rubilar y C. Gurstein, editores, Libro de Actas del I Simposio de Paleontología en Chile: 131-135.

Latorre, C., J.L. Betancourt, K.A. Rylander, J. Quade y O. Matthei 2003. A vegetation history from the arid prepuna of northern Chile (22-23 ${ }^{\circ}$ S) over the last 13,500 years. Palaeogeography, Palaeoclimatology, Palaeoecology 194: 223-246.

Linnaeus, C. 1758. Systema Naturae per Regna tria Naturae secundum Classes, Ordines, Genera, Species, cum Characteribus, Differentiis, Synonymis, Locis. Salvii, Stockholm.

López-Aranguren, D. 1930. Camélidos fósiles argentinos. Anales de la Sociedad Científica Argentina 109:15-55; 97-126.

López, P. y R. Labarca 2005. Macrauchenia (Litopterna), Hippidion (Perissodactyla), Camelidae y Edentata en Calama (II Región): Comentarios taxonómicos y tafonómicos. Noticiario Mensual del Museo Historia Natural, Chile 355:7-10.

Lund, P. W. 1842. Blik paa Brasiliens dyreverden für sidste Jordomvaeltning. Fjerde Afhandling: Fortsaettelse af Pattedryene 9:137-208.

Marín, J.C., B. Zapata, B.A. González, C. Bonacic, J.C Wheeler, C. Casey, M. Bruford, E. Palma, E. Poulin, M.A. Alliende y A. Spotorno 2007. Sistemática, taxonomía y domesticación de alpacas y llamas: nueva evidencia cromosómica y molecular. Revista Chilena de Historia Natural 80:121-140.

Massone, M. 2004 Los Cazadores después del Hielo. Colección de Antropología 7, Centro de Investigación Barros Arana. Ediciones de la Dirección de Archivos y Museos, Santiago.

Meadow, R. 1987. Techniques for comparing bone measurement data from small samples. Trabajo presentado al Northeastern Faunal Analysis Conference. Storrs,
Connecticut.

Menegaz, A. 2000. Los camélidos y cérvidos del cuaternario del sector bonaerense de la Región Pampeana. Facultad de Ciencias Naturales y Museo, Universidad Nacional de La Plata, La Plata, Argentina, 211 pp.

Menegaz, A. y Ortíz-Jaureguízar, E. 1995. Los Artiodáctilos. Alberdi, M.T., G. Leone, y E.P. Tonni, editores, Evolución biológica y climática de la Región Pampeana durante los últimos 5 millones de años. Un ensayo de correlación con el Mediterráneo occidental, pp. 311-337. Monografías, Museo Nacional de Ciencias Naturales, CSIC, Madrid.

Menegaz, A., E. Goin y E. Ortíz-Jaureguízar 1989. Análisis morfológico y morfométrico multivariado de los representantes fósiles y vivientes del género Lama (Artiodactyla, Camelidae). Sus implicancias sistemáticas, biogeográficas, ecológicas y biocronológicas. Ameghiniana 26(3-4):153172.

Mengoni-Goñalons, G. L. y H. D. Yacobaccio 2006. The domestication of South American camelids: a view from the South-Central Andes. Zeder, M.A., D. Bradley, E. Emshwiller y B. D. Smith, editores, Documenting Domestication: New Genetic and Archaeological Paradigms, pp. 228-244. University of California Press, Berkeley.

Miotti, L. y M. Salemme 1999. Biodiversity, taxonomic richness and specialists-generalists during Late Pleistocene/Early Holocene times in Pampa and Patagonia (Argentina, Southern South America). Quaternary International 53/54:53-68.

Molina, G. I. 1782. Saggio Sulle Storia Naturale del Chile. Bologna, Italia.

Müller, P.L.S. 1776. Erste Classe, Säugende Thiere. Des Ritters Carl von Linné vollständiges Naturalsystem nach der zwölften Lateinischen Ausgabe, 1773-1776, pp. 1-62.

Nami, H.G. y A. Menegaz 1991. Cueva del Medio: aportes para el conocimiento de la diversidad faunística hacia el Pleistoceno-Holoceno en la Patagonia Austral. Anales del Instituto de la Patagonia, Serie Ciencias Humanas 20:117-132.

Owen, R. 1839. Fossil Mammalia. Darwin, Ch., editor, The Zoology of the Voyage of H.M.S. Beagle Under the Command of Captain Fitzroy R.N., During the Years 1832 to 1836, pp. 13-111 + XXXII PI. Smith, Elder \& Co, Londres.

Paunero, R.S., M. Cueto, A. Frank, G. Ghidini, G. Rosales, F. Skarbun 2004. Comunicación sobre campaña arqueológica 2002 en localidad La María, Santa Cruz. Civalero, M.T., P.M. Fernández, A.G. Guraich, editores, Contra viento y marea: arqueología de Patagonia, pp. 797-808. Instituto Nacional de Antropología, Buenos Aires. 
Prieto, A. y J. Canto. 1997. Presencia de un lamoide atípico en cueva Lago Sofía 4 (Última Esperanza) y Tres Arroyos (Tierra del Fuego) región de Magallanes, Chile. Anales del Instituto de la Patagonia, Serie Ciencias Humanas 25:147-150.

Roth, S. 1899. Descripción de los restos encontrados en la Caverna de Última Esperanza. Revista del Museo de La Plata 9:421-453.

Saxon, E. 1976. La Prehistoria de Fuego-Patagonia: Colonización de un hábitat marginal. Anales del Instituto de la Patagonia, Serie Ciencias Humanas 7: 63-73.

Scherer, C.S., J. Ferigolo, A.M. Ribeiro y C. Cartelle 2007. Contribution to the knowledge of Hemiauchenia paradoxa (Artiodactyla, Camelidae) from the Pleistocene of southern Brazil. Revista brasileira de paleontología 10(1):35-52.

Sylvestre, F., M. Servant, S. Servant-Vildary, C. Causse y M. Fournier 1999. Lake-level chronology in the south Bolivian Altiplano $\left(18-23^{\circ} \mathrm{S}\right)$ during Late-Glacial and early Holocene times based on radiocarbon, U/Th ages and diatom studies. Quaternary Research 51: 54-66.

Tauber, A. 1999. Hallazgo de una vicuña en el Pleistoceno de la Provincia de Córdoba. Ameghiniana 36(1)55-62. von den Driesch, A. 1976. A guide to the measurement of animal bones from archaeological sites. Harvard University, Peabody Museum of Archaeology and Ethnology, Peabody Museum Bulletin 1.

Weinstock, J., B. Shapiro, A. Prieto, J. C. Marín, B. A. González, M. Thomas, P. Gilbert y E. Willerslev 2009. The Late Pleistocene distribution of vicuñas (Vicugna vicugna) and the "extinction" of the gracile llama ("Lama gracilis"): New molecular data. Quaternary Science Reviews 28(15-16):1369-1373.

Wellman, R. 1972. Origen de la Cueva del Mylodon en Última Esperanza. Anales del Instituto de la Patagonia 3:97-101.

Wheeler, J.C. 1995. Evolution and Present Situation of the South-American Camelidae. Biological Journal of the Linnean Society 52:271-295.

Wheeler, J.C. 2006. Historia natural de la vicuña. Vilá, B., editor, Investigación, conservación y manejo de la vicuña, pp. 25-36. Proyecto MACS, Buenos Aires.

Villa-Martínez, R. y P. Moreno. 2007. Pollen evidence for variations in the southern margin of the westerly winds in SW Patagonia over the last 12.600 years. Quaternary Research 68: 400-409.

Anexo 1. Análisis de Componentes principales. Contribución de cada variable en los dos primeros componentes principales.

\begin{tabular}{lcc}
\hline Medida & C1 & C2 \\
\hline Gl & $-0,9779$ & 0,05487 \\
Bp & $-0,9786$ & $-0,1555$ \\
BFp & $-0,9859$ & $-0,131$ \\
Dp & $-0,9893$ & $-0,06381$ \\
DFp & $-0,9874$ & $-0,03087$ \\
Bd & $-0,9848$ & $-0,01602$ \\
SD & $-0,9277$ & 0,3633 \\
\hline
\end{tabular}

\begin{tabular}{lcc}
\hline Medida & C1 & C2 \\
\hline Gl & 0,9652 & $-0,1785$ \\
Bp & 0,9936 & 0,04913 \\
BFp & 0,9907 & $-0,001363$ \\
Dp & 0,9877 & $-0,09648$ \\
DFp & 0,9664 & $-0,1629$ \\
Bd & 0,9776 & 0,1348 \\
SD & 0,9602 & 0,256 \\
\hline
\end{tabular}

Tabla 3. Segunda falange posterior

\begin{tabular}{lcc}
\hline Medida & C1 & C2 \\
\hline GD & $-0,9877$ & $-0,0404$ \\
GB & $-0,9809$ & $-0,1665$ \\
GH & $-0,9768$ & 0,208 \\
\hline
\end{tabular}

Tabla 5. Carpo ulnar

\begin{tabular}{lcc}
\hline Medida & C1 & C2 \\
\hline Gl & $-0,9965$ & 0,08267 \\
Bp & $-0,9597$ & $-0,2509$ \\
BFp & $-0,9725$ & $-0,2146$ \\
Dp & $-0,9752$ & $-0,1298$ \\
DFp & $-0,9794$ & $-0,1091$ \\
Bd & $-0,9781$ & $-0,1115$ \\
SD & $-0,9488$ & $-0,1925$ \\
\hline
\end{tabular}

Tabla 2. Primera falange posterior

\begin{tabular}{lcc}
\hline Medida & C1 & C2 \\
\hline GD & $-0,9875$ & 0,0851 \\
GB & $-0,9742$ & $-0,2247$ \\
GH & $-0,984$ & 0,1371 \\
\hline \multicolumn{3}{c}{ Tabla 4. Carpo radial }
\end{tabular}

\begin{tabular}{lcc}
\hline Medida & C1 & C2 \\
\hline GD & $-0,986$ & $-0,09519$ \\
GB & $-0,9806$ & $-0,1572$ \\
GH & $-0,9663$ & 0,2566 \\
\hline
\end{tabular}

Tabla 6 Cuarto tarsiano 
Anexo 2. Resultados de los eigenvalues y porcentaje del componente que explica de la varianza total

\begin{tabular}{ccc}
\hline & Eigenvalue & \% var \\
\hline C1 & 6,67006 & 95,287 \\
C2 & 0,181653 & 2,595 \\
\hline
\end{tabular}

Tabla 1. Primera falange anterior

\begin{tabular}{ccc}
\hline & Eigenvalue & \% var \\
\hline C1 & 6,68739 & 95,534 \\
C2 & 0,153818 & 2,1974 \\
\hline
\end{tabular}

Tabla 3. Segunda falange posterior

\begin{tabular}{ccc}
\hline & Eigenvalue & \% var \\
\hline C1 & 2,89165 & 96,388 \\
C2 & 0,072616 & 2,4205 \\
\hline \multicolumn{3}{c}{ Tabla 5. Carpo ulnar }
\end{tabular}

Tabla 5. Carpo ulnar

\begin{tabular}{ccc}
\hline & Eigenvalue & \% var \\
\hline C1 & 6,76257 & 96,608 \\
C2 & 0,0820938 & 1,1728 \\
\hline
\end{tabular}

\begin{tabular}{ccc}
\hline & Eigenvalue & \% var \\
\hline C1 & 2,89253 & 96,418 \\
C2 & 0,0765297 & 2,551 \\
\hline \multicolumn{3}{c}{ Tabla 4. Carpo radial }
\end{tabular}

\begin{tabular}{ccc}
\hline & Eigenvalue & \% var \\
\hline C1 & 2,86746 & 95,582 \\
C2 & 0,0996278 & 3,3209 \\
\hline \multicolumn{3}{c}{ Tabla 6. Cuarto tarsiano }
\end{tabular}

